\title{
Implementation of Acoustic Communication in Under Water Using BPSK
}

\author{
K.Saiteja ${ }^{1}$, K.Rahul kumar ${ }^{2}$, J.Vamsee kumar ${ }^{3}$, M.Gaganviharvarma ${ }^{4}$, \\ CH.Gayatri ${ }^{5}$ \\ 1,2,3,4,5 Department of ECE, LENDI Institute of Engineering and Technology, JNTU-Kakinada, India
}

\begin{abstract}
The aim of the project is to make a prototype of acoustic communication system. The acoustic channel introduces some challenges in form of multipath propagation causing attenuation of the signals and Inter Symbol Interference (ISI). A coherent underwater acoustic communication system must deal with the inter symbol interference caused by the time-varying, dispersive, shallow-water ocean environment. The system is characterized with high data rate and low bit error probability using BPSK modulation technique. This paper contains the time-reversal process to acoustic communications in order to improve data telemetry in the ocean. To combat the influence of the channel we used a linear adaptive equalizer. The code was implemented in MATLAB, using acoustic signals different intermediate outputs are simulated and modulation and demodulation of signals are obtained which are shown in this project.
\end{abstract}

Keywords: Acoustics communication, BPSK modulation, Inter Symbol Interference, Linear adaptive equalizer, Time reversal, Under Water communication.

\section{Introduction}

The underwater acoustic telemetry channel is band limited and reverberant which poses many obstacles to reliable and high-speed digital communications. Prior to the late 1970's, there were a few published attempts of acoustic modems. Analog systems were developed, which were essentially sophisticated loudspeakers, but they had no capability for mitigating the distortion introduced by the highly reverberant underwater channel. Paralleling the developments applied to the severely fading radio frequency atmospheric channels, the next generation of systems employed frequency-shift-keyed (FSK) modulation of digitally encoded data. As an energy-detection (incoherent) rather than phase-detection (coherent) algorithm, FSK systems were seen as intrinsically robust to the time and frequency spreading of the channel. The use of digital techniques was important in two respects. First, it allowed the use of explicit error-correction techniques to increase reliability of transmissions. Second, it permitted some level of compensation for the channel reverberation both in time (multipath) and frequency (Doppler spreading)[1]. The inefficient use of bandwidth of incoherent systems coupled with the limited availability of bandwidth underwater makes them ill-suited for high-data-rate applications except at short ranges. Larger data rate-range products required the use of coherent modulation. Communication channels may be coarsely divided into two categories according to the performance-limiting quantity ,power, or bandwidth.The early 1990's have yielded a plethora of published coherent systems that moved acoustic telemetry into the horizontal ocean channel. This is possible due to the use of a powerful receiver algorithm that coupled a decision feedback adaptive equalizer with a second-order phase-locked loop. Using quadrature phase-shift-keyed (QPSK) modulation, a data link of $1000 \mathrm{bit} / \mathrm{s}$ at $90 \mathrm{~km}$ was demonstrated[1].The underwater acoustic channel is a wireless communication medium. Absorption at high frequencies and ship noise at low frequencies limit the usable bandwidth. Acoustics is the scientific study of sound, especially of its generation, transmission, and reception. Acoustic means sound related, so acoustic communication would be any form of communication that uses sound.

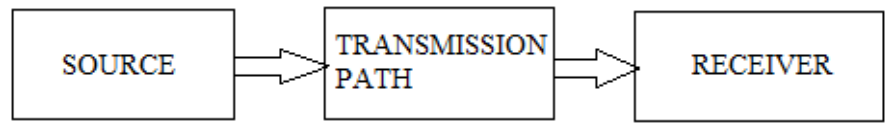

Fig.1.1: Communication system

The communication system we design should be robust against multipath propagation and coherent detection is preferable. To improve an ordinary system and to achieve this robustness we are to use two branch diversity and equalization. The system should enable a user to transmit a data file from one PC to another. The prototype should be able to transmit data with a low bit error probability and high average transmission rate. 
Higher bit rate causes more ISI and therefore increases the necessary signal processing. Since the 1990s, various investigations on decision feedback equalizers DFEs and time reversal approaches have contributed to the advancement of coherent underwater acoustic communications. It has been shown that the DFE coupled with a phase tracking process presents a practical solution to the multi-path spread and fast phase fluctuation of underwater acoustic channels. The DFE with joint phase tracking can be extended to multiple hydrophone channels to form a multichannel DFE. In the multichannel DFE, the total number of adaptive feed forward taps increases with the number of channels. The implementation complexity of the multichannel DFE is high for a moderate to large number of hydrophone channels, which often are needed to achieve reliable performance in dynamic ocean environments. In order to alleviate the complexity issue, channel estimation can be incorporated into the DFE structure [2]. The study of broadband acoustic wave propagation in shallow water in the presence of in homogeneities has attracted increasing attention in recent years, with one of the more important sub-topics being the study of the intensity fluctuations of the acoustic field due to nonlinear internal waves. In particular, the frequency dependence of the variability of sound propagation in shallow water is of interest and is closely related to the spatial and temporal variability of physical oceanographic entities such as internal waves [3].

\section{Choice Of Modulation Method}

With the fast development of modern communication techniques, the demand for reliable high date rate transmission is increased significantly, which stimulate much interest in modulation techniques. Different modulation techniques allow you to send different bits per symbol and thus achieve different throughputs or efficiencies. The modulated signals are then demodulated at the receiver where the original digital message can be recovered. There is also a requirement for estimating two different carrier phases in the BFSK receiver which makes coherent demodulation complex and impractical. BPSK with coherent detection perform better than coherent BFSK for all signal to noise ratios. This is of course sounds like a very pleasant property when making the choice of modulation technique. Binary PSK imposes the information on the phase of the carrier. It is clear that the information maps the signal constellation positive and negative amplitudes respectively. Clearly this is a linear modulation scheme and therefore it will be possible to design a linear MMSE equalizer to combat the ISI. We therefore choose BPSK as the modulation scheme in our communication system.

\section{Bpsk Technique}

BPSK is a digital modulation technique that separates bits by shifting the carrier 180 degrees. A carrier frequency signal is chosen that is known by both the transmitter and the receiver. Each bit is encoded as a phase shift in the carrier at some predetermined period. When a 0 is sent, the carrier is transmitted with no phase shift, and when a 1 is sent, the carrier is phase-shifted by 180 degrees. When a sequence is given which changes the value from a 0 to a 1 or from a 1 to a 0 , a change of phase is indicated in the positive and negative y axis. Digital modulation technique is classified into two types coherent and Non coherent techniques depending on the phase recovery circuit. The phase recovery ensures that the oscillator supplying the local generated carrier wave in the receiver is synchronized (in both frequency and phase) to the oscillator supplying carrier wave used to originally modulate the incoming data stream in the transmitter. The output waveform shown below for the logic 0 and 1 is shown below for the 0 degrees and 180 degrees.
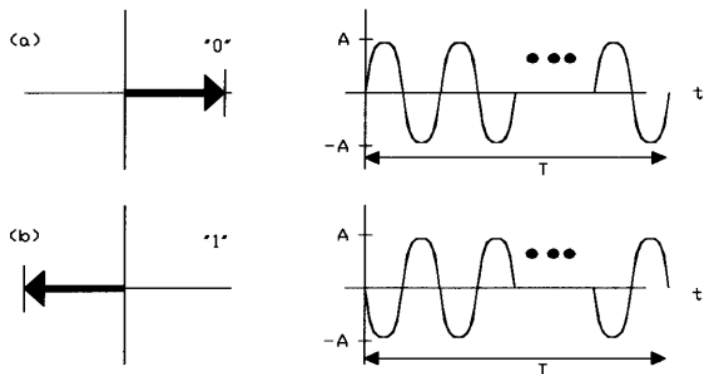

Fig.3.1(a) \& (b): Logic 0\&1

For logical 0 the phasor diagram is shown along the positive axis and logical 1 the phasor diagram is shown along the negative axis. 


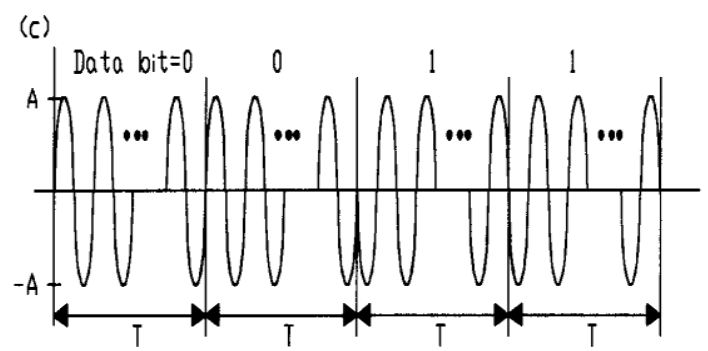

Fig.3.2: Phasor Diagram

BPSK modulation technique the constellation diagram shown below which are transmitting binary data there are only two points. As these two points are at equal distance from origin we would expect them to represent equal magnitude carriers.

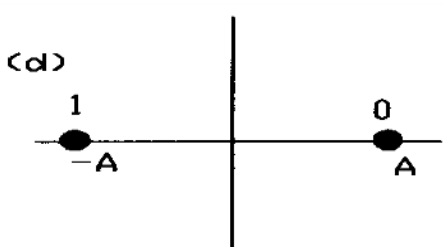

Fig.3.3: Bpsk Technique

In Binary Phase shift keying (BPSK), determines the phase of transmitted signal and decoding the transmitted information. This problem is also known as the carrier recovery attempt is made to recover the phase of the carrier. When a phase point at, say, 180 degrees is selected to reflect the information being transmitted, the phase of the transmitted carrier is set to 180 degrees. However the phase of the carrier is often changed by the transmission channel with result that the receiver measure a different phase. In order to overcome this problem there are two ways.

1. Coherent detection,

2. Non coherent detection.

In coherent detection we have to measure the phase change imposed by the channel and then the receiver determine the transmitted phase. This is known as the coherent detection.

In Non coherent detection we have transmit the phase difference rather than actual phase. The receiver merely compares the actual phase with current phase and the phase changed is removed. This is known as Non coherent detection.

In BPSK the signal bandwidth for the communications channel needed depends on the symbol rate, not on the bit rate. In this symbol rate is equal to bit rate because only two bits are transmitted. The actual formula for the symbol rate is given as:

Symbol rate $=($ bit rate $) /($ The number of bits transmitted with each symbol $)$

\section{Time Reversal}

A Time-reversal mirror TRM can therefore be realized with a source-receiver array. The incident signal is received, time reversed, and transmitted from sources contiguous with the receiving hydrophones [4].The application of time-reversal methods (phase conjugation in the frequency domain) for underwater acoustic communications has already been suggested and some calculations for a $3500-\mathrm{Hz}$ pulse with a kilohertz bandwidth has demonstrated the temporal multipath recombination and side lobe suppression needed for underwater communications [5]. Phase conjugation is an environmentally self-adaptive process which may therefore have significant applications to localization and communications in complicated ocean environments [4].Adaptive channel equalization has been used in coherent underwater acoustic communication systems to deal with the inter-symbol interference (ISI) caused by time varying dispersive multipath environments . Time reversal is an approach that can be used independently of or in conjunction with adaptive channel equalization $[5]$.

\subsection{Transmitter:}

\section{Model Of The Communication System}

In Matlab the main blocks to consider in the transmitter is packing the data according to the transmission protocol and modulation of the signal. A program for converting a data file into a bit sequence was not done in the Matlab implementation. The original sequence, i.e. the bitmap picture, is read as 0 and 1 but 
those are mapped to represent the phase shift made by the BPSK modulation scheme. A simple model of the transmitting side of the system could be seen in the following block diagram.

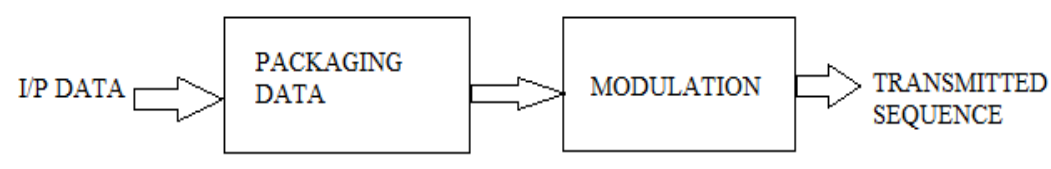

Fig.5.1: Block diagram of transmitter.

\subsubsection{Packing Data:}

The bit sequence then has to be packed according to our transmission protocol so that the receiver understands the received sequence. The protocol is a block oriented transmission protocol. In the initial stage information that is used to calibrate the receiver is sent. The Power burst is simply some equi-probable bits that are sent so that the receiver can detect an increase in signal power. After that, a synchronization sequence is sent which is known both by the receiver and transmitter. Then a guard sequence is sent, this is to a room for possible corrections to achieve precise synchronization. After that Block 0 is sent. This block is for training the equalizer coefficients. Optimizing the system will then cause the error probability to decrease and of course increase the robustness for inter symbol interference.

\subsection{Receiver:}

When designing the Matlab implementation of the receiver, five blocks of importance have to be considered. These are demodulation of the received signal synchronization, equalization, diversity combination and detection.

A simple block diagram of the receiver is depicted in figure below.

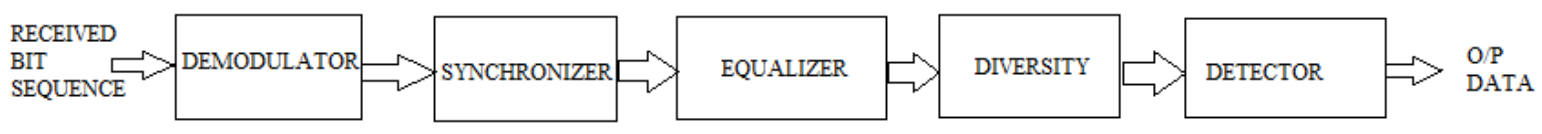

Fig.5.2: Block diagram of receiver.

When receiving the transmitted sequence for the Matlab implementation, this is done manually. The received data is then saved in two vectors, one for the left channel and one for the right channel. These vectors are then available for signal processing in our receiver function.

\subsubsection{Demodulation}

The signal processing for the left and right channel is done separately all the way to the diversity combination just before the detection. Therefore only data from one branch will be considered in the discussion of the signal processing.

\subsubsection{Synchronization}

When receiving a transmitted file the sampling does not start exactly when the real signal starts. For the receiver to know when the desired data starts we first need to find an approximate synchronization. After that we need a precise synchronization that synchronizes the receiver to the exact sample where the data starts in the received sequence.

\subsubsection{Equalizer}

An equalizer equalizes the received distorted signal, this has to be employed before any detection of the received symbols. The equalizer removes (attenuates) these older symbols by choosing the filter taps so that the taps that covers the older symbols (the unwanted symbols at the current sampling instant) are assigned very low values resulting in a strong attenuation of these symbols, while the currently transmitted data symbol passes the equalizer almost unaffected. In this way, we minimize the effect of the unwanted symbols on the decision variable that is created at the current sampling instant.

The equalizer also uses a sort of decision feedback to update the filter taps. In this decision feedback, the assumed correctly detected bits (zeros and ones) are fed back to a summator, which computes the difference between the nth decision variable (nth output from the equalizer) and the nth detected bit. 


\subsubsection{Diversity}

To increase the performance of the communication system, i.e. to obtain a lower bit error probability $\mathrm{P}_{\mathrm{e}}$ a two-branch space diversity with a sort of simplified maximal ratio combination was implemented. For practical reasons the diversity is implemented in the equalizer. The two-branch space diversity is obtained in the receiver, using two signals from each other spaced microphones to receive the transmitted signal. When the two received signals enter the equalizer, they are both processed (equalized).

Before any diversity combining of the two signals can take place, they have to be co-phased, i.e. processed so that they are in phase relative to each other. This co-phasing process is taken care of by the equalizer. When the two signals are in phase, a combining of the two signals should take place in order to obtain one new signal that has a signal to noise ratio (SNR) that is greater than what the two signals have separately. To get knowledge in how to combine the two signals, the error

$$
e_{i}(n)=r_{d}(n)-d^{\wedge}(n) \quad \text { for } i=1,2
$$

is then computed for each branch. The errors $e_{1}$ and $e_{2}$ are then compared and the branch with the greatest error is lowest weighted in the summation of the two signals that follows in order to create the input signal to the detector (the decision variable). In this way a diversity gain is achieved, i.e. we obtain a signal (the input signal to the detector) with a greater SNR than the signals in each branch have separately and thereby a lower bit error probability $\mathrm{P}_{\mathrm{e}}$.

\subsubsection{The Detector}

The input to the detector is the sum of the two equalized signals from the two branches denoted by $r_{d}(n)$. The detector simply controls the sign on the real part of $r_{d}(n)$. If the real part of $r_{d}(n)$ is greater than zero the detector decides that a zero was transmitted and if it is less than zero the detector decides that a one was sent.

\section{Results}

Before we summarize the results of the numerous Matlab simulations that have been made to optimize the communication system, we shortly repeat the requirements and goals that were set up for the communication system before the project started. After having optimized the system parameters to achieve as high data rate $\mathrm{R}$ as possible and still maintain as low bit error probability $\mathrm{P}_{\mathrm{e}}$ as possible, the following results were obtained for communication with a spacing of 3 meters between the speaker and the microphones. Simulations are carried out on implementation different techniques and obtained results for them in matlab. In matlab simulations are done using BPSK techniques. Results for BPSK modulation/demodulation technique for random bit stream in MATLAB are also shown. The plots show the transmitted and received waveform of the BPSK technique.

This plot explains about the base-band signal spectrum in frequency domain where the entire information is present in obtained output.

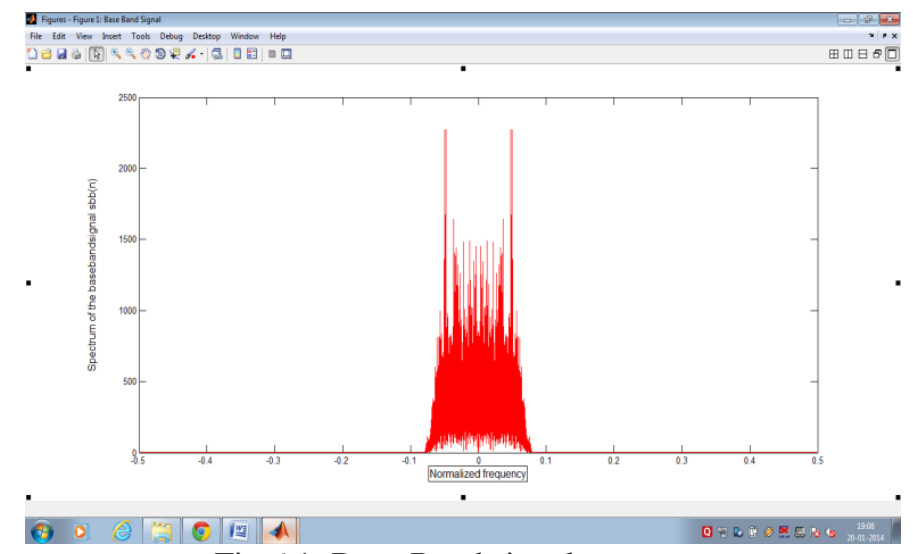

Fig.6.1: Base-Band signal spectrum

This plot explains about the pulse shaped signal before modulation. 


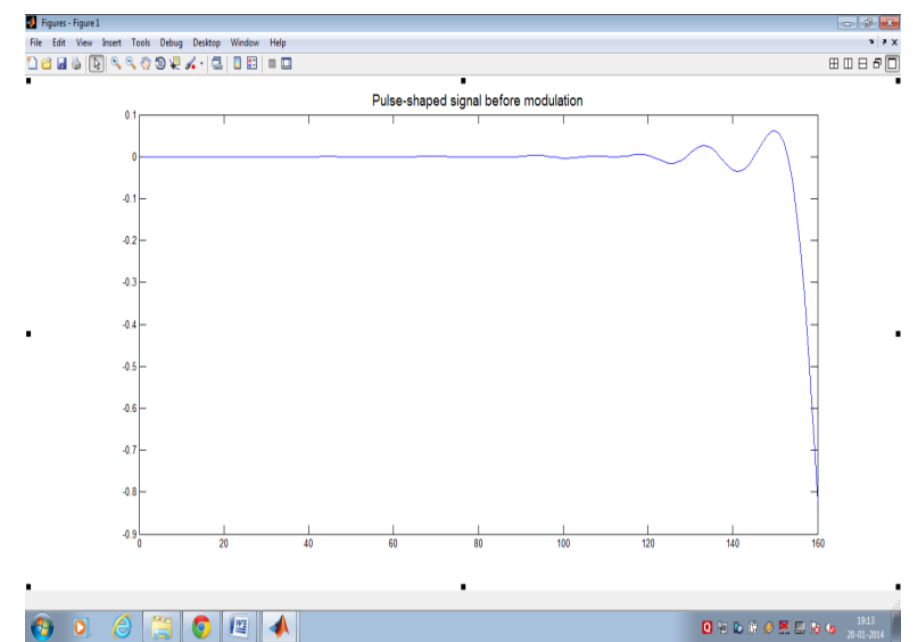

Fig.6.2: Pulse-shaped signal

This plot explain about the signal which is obtained after it is sent from the BPSK technique where the transmitter signal obtained whch is sent towards the channel.

The Red colour plot explain about the spectrum of BPSK modulated signal in frequency domain.

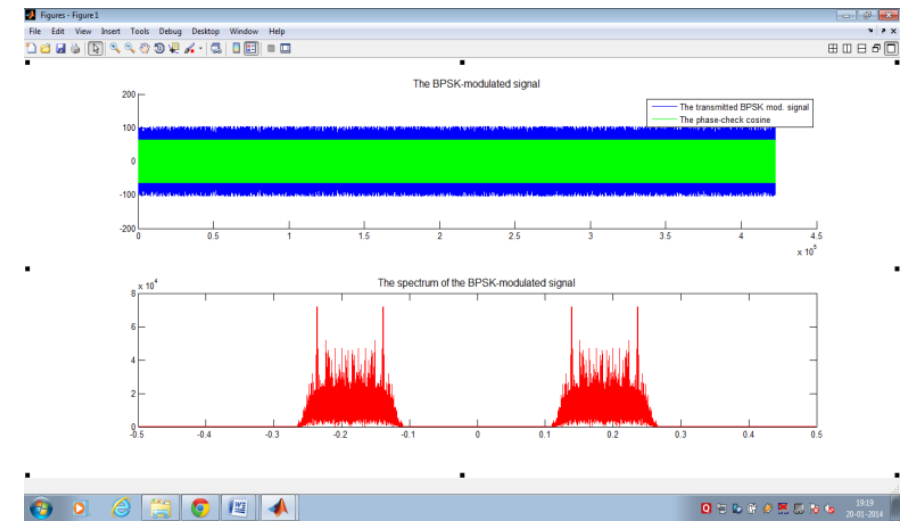

Fig.6.3: Modulated signal in BPSK technique

This plot explains about the noise signal.

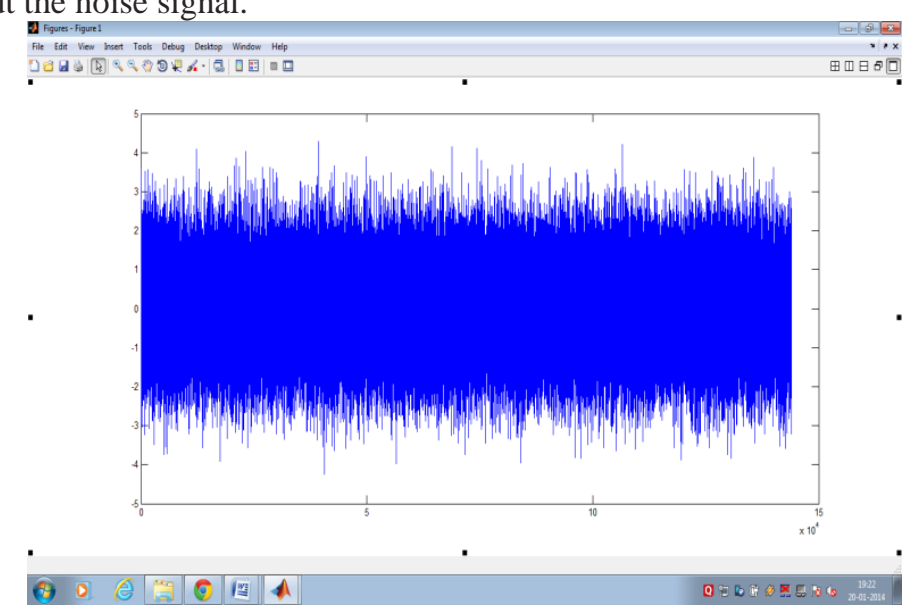

Fig.6.4: Noise signal

This plot explains about the in-phase and quadrature phase BPSK constellation diagram where the decision region is shown. 


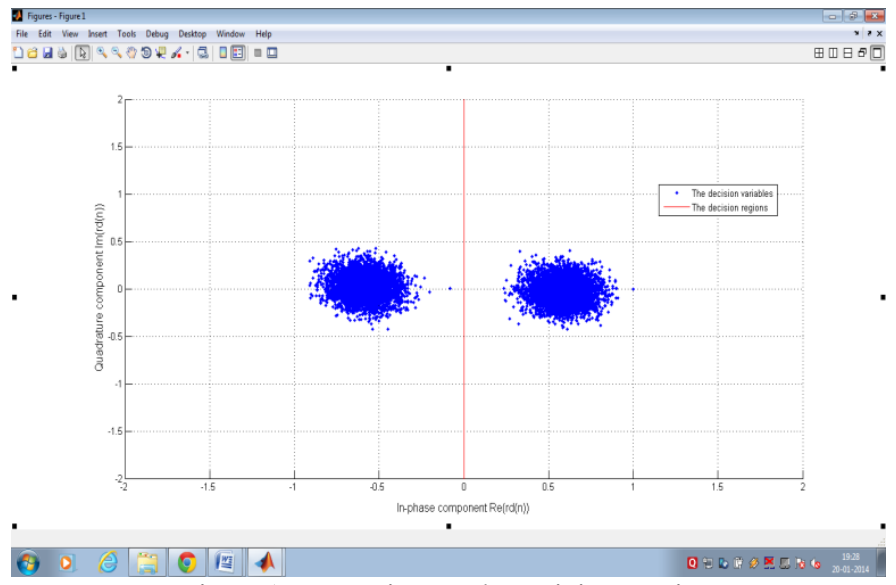

Fig.6.5: Detection and Decision region

This plot explains about the demodulated un-equalized signal where the signal output has un-equalized frequencies which are shown in the first plot.

In the next plot the demodulated un-equalized signal output is made in the form of equalized frequencies and shown.

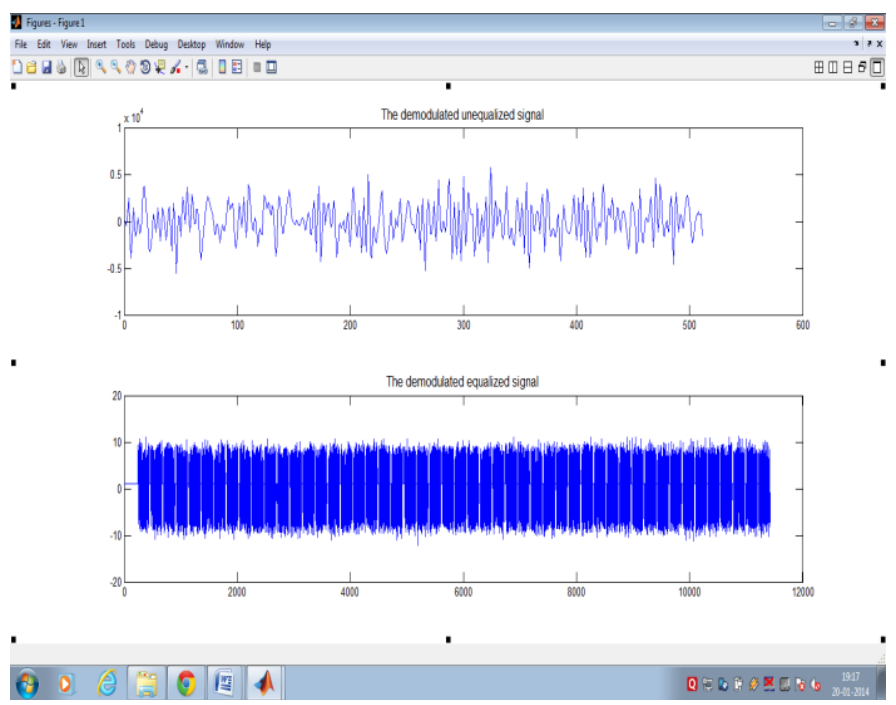

Fig.6.6: Un-equalized and Equalized Demodulated signal

This plot explains the signal decision region during detected data sequence where the decision region has the signal output near origin. This makes that the signal with No errors.

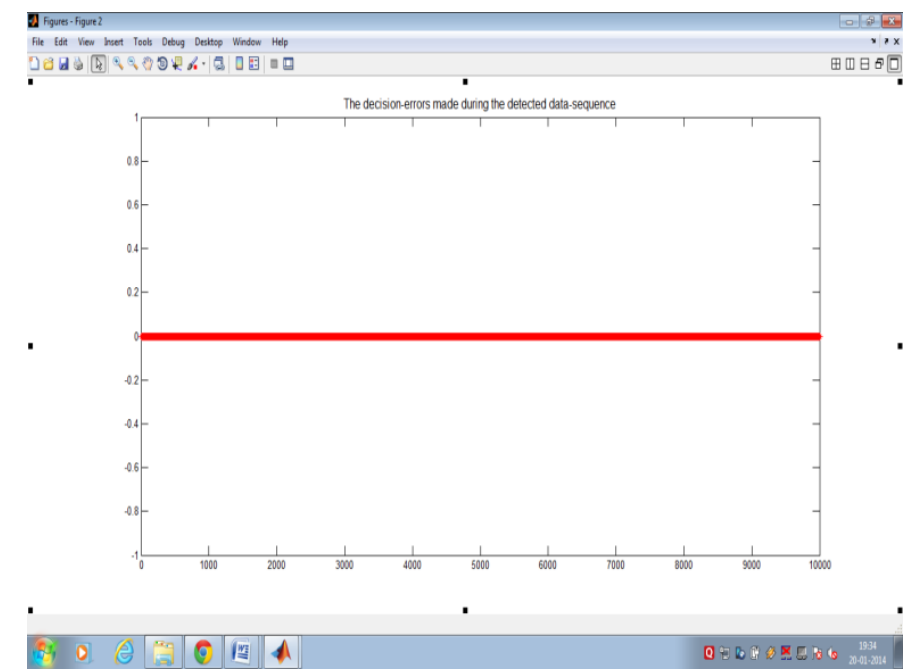


Fig.6.7: Decision and Detection region

This plot explains about the equalizer coefficients near branch 1, 2 for filters where we use low pass filters.

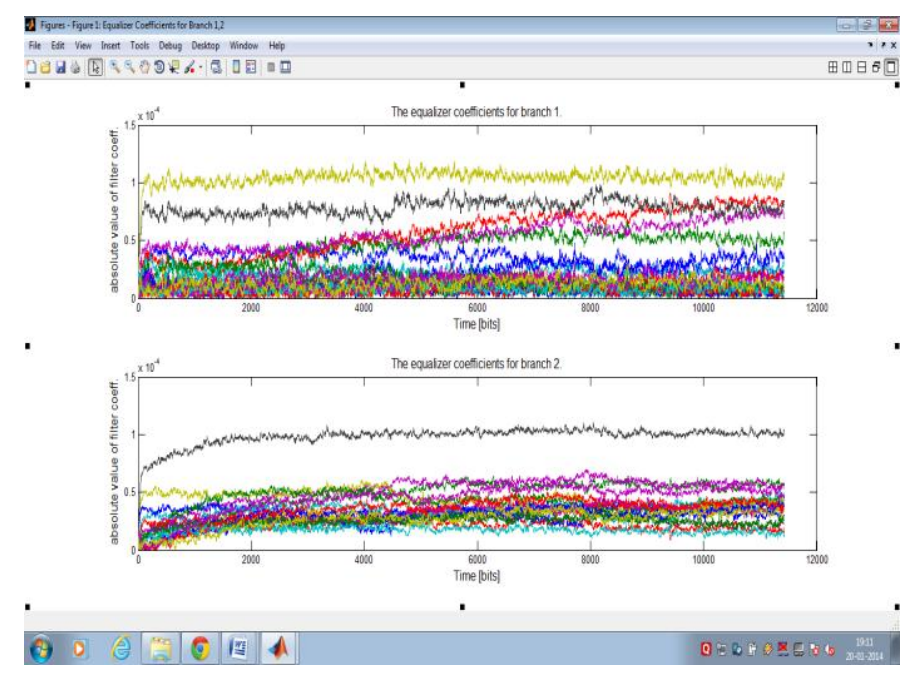

Fig.6.8: Equalizer Coefficients for Branch 1,2

This plot explain the equalizer coefficients near branch 1,2 where the filter coefficients are made without errors.

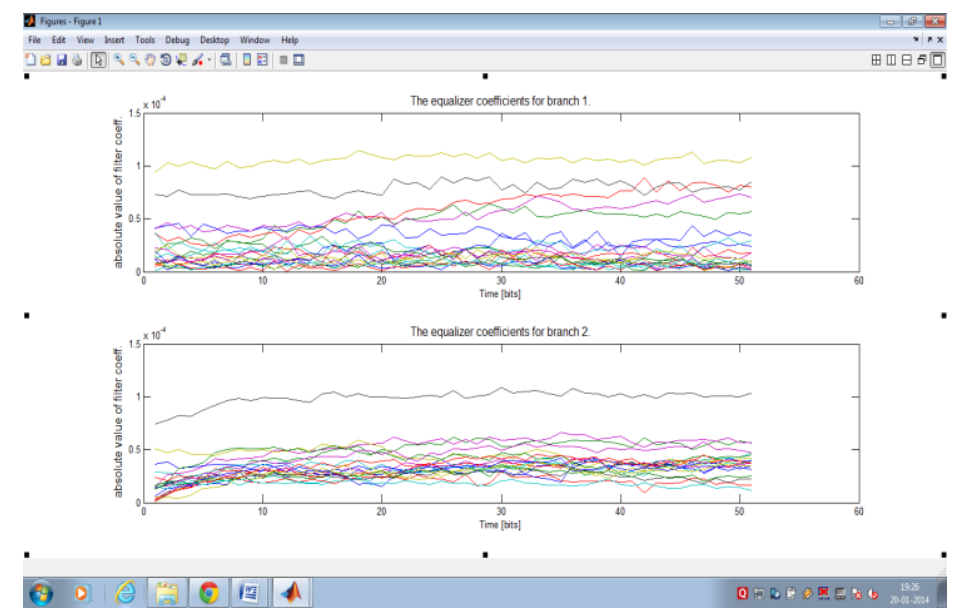

Fig.6.9: Equalizer coefficient signal

As can be seen from the results above, by using diversity we obtain a pretty large diversity, gain and therefore the bit error probability $\mathrm{P}_{\mathrm{e}}$ can be decreased with $4 \%$ and there by the goals that were set for the communication system are achieved. 


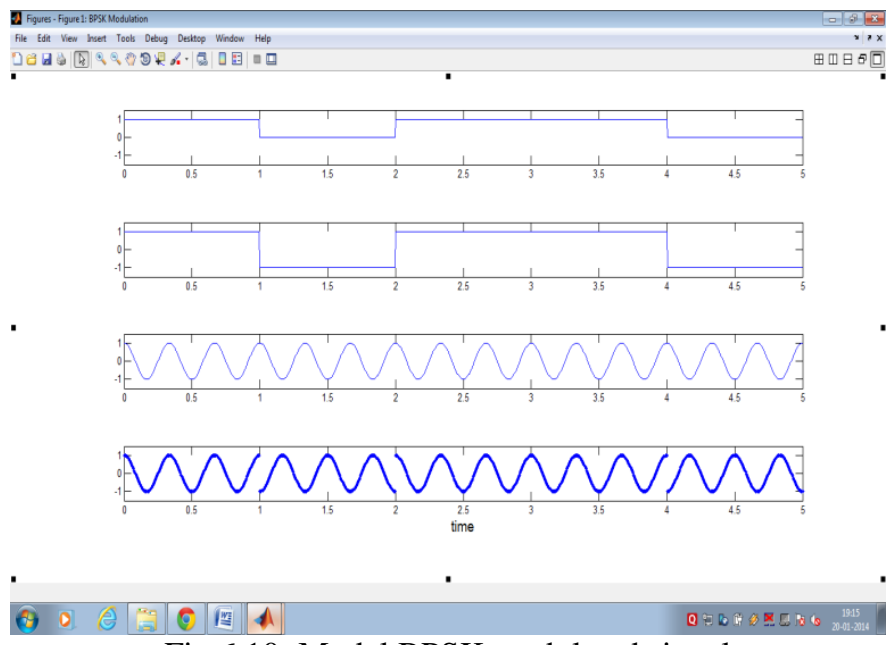

Fig.6.10: Model BPSK modulated signal

\section{Future Scope}

Under water communication finds application in many fields like oceanographic data collection, pollution, monitoring, off shore exploration, disaster prevention. The enabling technology for these applications is acoustic wireless networking. In order to increase the bandwidth of the modulation technique so that more number of bits are transmitted, we will use QAM modulation technique instead of BPSK or The performance analysis of BPSK modulation will be further increased with the addition of IDMA scheme along with BPSK technique.

\section{Conclusion}

In this work, both the downstream and upstream directions of the signal is done. First we aimed to maximize the data rate and then optimize the rest of the system to obtain an error probability below $1-2 \%$. The matlab simulations explain about the signal transmission using BPSK technique and data rate of the signal sent towards the receiver. In the receiver section explain about the signal bit rate and false detection region of the signal whether the signal obtained towards the receiver should be without errors. In this way the acoustic signal transmission and reception takes place.

\section{Acknowledgement:}

We, the authors thank the Department of ECE, LENDI Institute of Engineering and Technology for their continuous support in completing this paper.

\section{References}

[1] D. B. Kilfoyle and A. B. Baggeroer, "The state of the art in underwater acoustic telemetry," IEEE J. Ocean. Eng., vol. 25, no. 1, pp. 4-27, Jan.2000.

[2] A. Song, M. Badiey, H.-C. Song, W. S. Hodgkiss, M. B. Porter,and the KauaiEx Group, "Impact of ocean variability on coherentunderwater acoustic communications during the Kauai experiment(KauaiEx)," J. Acoust. Soc. Amer., vol. 123, no. 2, pp. 856-865,Feb. 2008.

[3] M. Badiey, B. G. Katsnelson, J. F. Lynch, and S. Pereselkov, "Frequencydependence and intensity fluctuations due to shallow water internalwaves," J. Acoust. Soc. Amer., vol. 122, no. 2, pp. 747-760, Aug.2007.

[4] W. A. Kuperman, W. S. Hodgkiss, H. C. Song, T. Akal, C. Ferla, andD. R. Jackson, "Phase conjugation in the ocean: Experimental demonstrationof an acoustic time-reversal mirror," J. Acoust. Soc. Amer., vol.103, no. 1, pp. 25-40, Jan. 1998.

[5] G. F. Edelmann, T. Akal, W. S. Hodgkiss, S. Kim, W. A. Kuperman,and H. C. Song, "An initial demonstration of underwater acoustic communicationsusing time reversal," IEEE J. Ocean. Eng., vol. 31, no. 3,pp. 602-609, Jul. 2002.

[6] J. Gomes, A. Silva, and S. Jesus, "Adaptive spatial combining for passivetime-reversed communications," J. Acoust. Soc. Amer., vol. 124,no. 2, pp. 1038-1053, Aug. 2008.

[7] H. C. Song and S. M. Kim, "Retrofocusing techniques in a waveguidefor acoustic communications (L)," J. Acoust. Soc. Amer., vol. 121, no.6, pp. 3277-3279, Jun. 2007.

[8] Passive Time Reversal Acoustic CommunicationsThrough Shallow-Water Internal Waves"Aijun Song, Member, IEEE, Mohsen Badiey, Member, IEEE, Arthur E. Newhall, Member, IEEE,James F. Lynch, Fellow, IEEE, Harry A. DeFerrari, and Boris G. Katsnelson., IEEE JOURNAL OF OCEANIC ENGINEERING, VOL. 35, NO. 4, OCTOBER 2010

[9] Modulation and Demodulation Techniques for Underwater Acoustic CommunicationsJohn. G. Proakis, University of California, San Diego,jproakis@ucsd.edu

[10] Underwater Acoustic Communications Using Time ReversalG. F. Edelmann, H. C. Song, Member, IEEE, S. Kim, W. S. Hodgkiss, Member, IEEE, W. A. Kuperman, and T. Akal. 